\title{
RISK OF COVID-19 INFECTION AT WORK AND PSYCHO-PHYSICAL STRAIN: THE MODERATING ROLE OF NEGATIVE AFFECTIVITY
}

\author{
Damiano Girardi ${ }^{1}$, Alessandro De Carlo ${ }^{2}$, Laura Dal Corso ${ }^{1}$, Annamaria Di Sipio ${ }^{1}$, \\ \& Alessandra Falco ${ }^{1}$ \\ ${ }^{I}$ FISPPA Section of Applied Psychology, University of Padua, Padua (Italy) \\ ${ }^{2}$ Preventive Medicine and Hygiene, Department of Cardiac, Thoracic, Vascular Sciences and Public \\ Health, University of Padua, Padua (Italy)
}

\begin{abstract}
The ongoing outbreak of COVID-19 is severely affecting many areas of everyday life, including family, education, and work. Hence, safety at work - both physical and psychological - plays a central role for organizations, which need to be productive while, at the same time, preserving employees' health and well-being. Building on the job demands-resources (JD-R) model applied to safety at work, in this study we proposed that the perceived risk of being infected with COVID-19 at work can be conceptualized as a job demand (i.e., a risk factor for work-related stress). We also proposed that negative affectivity (i.e., a dispositional dimension that reflects pervasive individual differences in negative emotionality and self-concept; NA) may be conceived as a personal demand, that is, an individual characteristic that hinders employees' abilities to effectively cope with their work environment. Hence, according to the health impairment process of the JD-R, in this study we hypothesized that the perceived risk of being infected at work (PRIW) is positively associated with psycho-physical strain (i.e., stress-related psycho-physical symptoms), which, in its turn, is negatively associate with employees' job performance. We also hypothesized that NA affects the association between PRIW and psycho-physical strain, which is expected to be stronger for high-NA individuals. The study was carried out on a sample of 353 workers who completed a self-report questionnaire aimed at determining PRIW, NA, psycho-physical strain, and job performance. Data were analyzed using path analysis. Results showed that PRIW was positively associated with psycho-physical strain, which, in its turn, was negatively associate with job performance. The association between PRIW and job performance was not significant. Interestingly, NA moderated the association between PRIW and psycho-physical strain, which was stronger for high-NA individuals, and not significant for low-NA individuals. Overall, this study suggests that the JD-R can be successfully applied to safety at work during the ongoing outbreak of COVID-19. In line with the JD-R, PRIW (a job demand) was negative associated with workers' health and job performance, although indirectly in the latter case. Moreover, NA (a personal demand) exacerbated the association between PRIW and psycho-physical strain. From a practical standpoint, this study suggests that organizations should provide workers with adequate job resources to manage the risk of infection and achieve their objectives safely (i.e., primary prevention). Furthermore, in terms of secondary prevention, interventions could help high-NA employees to develop skills to cope effectively with the risk of infection at work.
\end{abstract}

Keywords: COVID-19, perceived risk, negative affectivity, psycho-physical strain, job performance.

\section{Introduction}

To date, the COVID-19 pandemic has afflicted tens of millions of people worldwide, with serious health, social, and economic consequences. With respect to the work context, employees from several occupational sectors are now facing the risk of being infected with COVID-19 at work. Indeed, workplaces and work activities have some characteristics that may facilitate the spread of SARS-CoV-2 (e.g., physical proximity, frequent social interactions). Hence, safety at work plays a key role for organizations, which strive to be productive while, at the same time, preserving employees' health and well-being. Interestingly, recent research suggests that physical and psychosocial safety need to be considered jointly (Yaris, Ditchburn, Curtis, \& Brook, 2020). With respect to the COVID-19 outbreak, this implies that work-related factors that may lead to both physical and psychological outcomes related to COVID-19 (e.g., contracting COVID-19 and work-related stress/job burnout, respectively) need to be 
thoroughly considered. Building on the job demands-resources model (JD-R; Bakker \& Demerouti, 2017) applied to safety at work (Nahrgang, Morgeson, \& Hofmann, 2011), in this study we propose that the perceived risk of being infected with COVID-19 at work (PRIW) can be conceptualized as a job demand, that is, a risk factor for work-related stress. We also propose that negative affectivity (NA; Watson \& Clark, 1984) can be conceived as a personal demand that moderates the association between PRIW and work-related stress, which is expected to be stronger when NA was high.

\subsection{The perceived risk of being infected at work and the JD-R model}

The JD-R is a flexible theoretical model that has been applied to several work-related areas, including occupational stress and well-being (Schaufeli \& Bakker, 2004), work-home interference (De Carlo, Girardi, Falco, Dal Corso, \& Di Sipio, 2019), and also safety at work (Nahrgang et al., 2011). According to the JD-R model, job demands are those aspects of the job that require sustained effort (i.e., physical and/or psychological), and are therefore associated with certain costs (i.e., physiological and/or psychological; Bakker \& Demerouti, 2017). In line with the health impairment process of the JD-R, high levels of job demands require efforts and exhaust employees' mental and physical energies. Over time, this may lead to health problems, for example in terms of psycho-physical strain (i.e., stress-related psycho-physical symptoms), and negative organizational outcomes, including reduced job performance.

With respect to workplace safety, job demands may include risks and hazards or physical demands, whereas consequences encompass psycho-physical symptoms and safety outcomes (e.g., safety-related work behaviors, accidents, and injuries; Nahrgang et al., 2011; Yaris et a., 2020). In line with the transactional models of stress applied to the JD-R (Crawford, LePine, \& Rich, 2010), which emphasize the central role of individual appraisals in the stress process, in this study we conceptualized the perceived risk of infection at work as a job demand. Risk perception has been defined as "subjective assessment of the probability of a specified type of accident happening and how concerned we are with such an event" (Marek, Tangernes, \& Hellesøy, 1985, p.152). Accordingly, a cognitive and an emotional dimension of risk perception have been identified in the literature (Rundmo \& Iversen, 2004). In line with the definition of job demands and the health impairment process of the JD-R, PRIW requires effort expenditure at work (e.g., to manage risks), thus depleting workers' mental and physical resources (e.g., time and energies). Over time, this may lead to the onset of psychological and physical symptoms (i.e., psycho-physical strain). Hence, we hypothesized PRIW to be positively associated with psycho-physical strain.

Hypothesis 1 (H1): PRIW is positively associated with psycho-physical strain.

Furthermore, in line with the JD-R, workers with high levels of health complaints do not have adequate levels of resources (e.g., cognitive or energetic) to reach their goals at work (Bakker $\&$ Demerouti, 2017). Accordingly, we hypothesized that psycho-physical strain is negatively associate with employees' job performance. performance.

Hypothesis 2 (H2): Psycho-physical strain is negatively associated with employees' job

\subsection{The moderating role of negative affectivity}

In the context of the JD-R model, personal demands have been recently defined as those characteristics of the self that compel individuals to invest exaggerated effort in their work and/or prevent them to cope successfully with their work environment, thus being associated with psychological and/or physical costs (Zeijen, Brenninkmeijer, Peeters, \& Mastenbroek, 2021). In this study, we propose that negative affectivity (NA; Watson \& Clark, 1984) may be conceived as a personal demand. NA is defined as a dispositional dimension that reflects pervasive individual differences in negative emotionality and self-concept (Watson \& Clark, 1984). Previous empirical research has shown that NA may affect both the choice as well as the effectiveness of coping strategies, with NA being associated with less beneficial coping styles (Eaton \& Bradley, 2008; Bolger \& Zuckerman, 1995). Hence, we hypothesized NA to moderate the association between PRIW and psycho-physical strain, with this association being stronger when NA was high.

Hypothesis 3 (H3): NA moderates the association between PRIW and psycho-physical strain.

Overall, based on the JD-R and the aforementioned arguments, we hypothesized that PRIW has a negative indirect effect on job performance through psycho-physical strain, with this indirect effect being moderated by NA (i.e., conditional indirect effect; Preacher, Rucker, \& Hayes, 2007). More specifically, we expected this negative indirect effect to be stronger when NA was high.

Hypothesis 4 (H4): The indirect effect of PRIW on job performance is moderated by NA. 


\section{Methods}

The study was conducted among workers from different organizations in Italy $(N=353)$. The sample consisted of 180 women and 172 men (one missing values), with a mean age of 36.4 years $(S D=12.3)$. Participants completed an online, self-report questionnaire aimed at determining job performance, psycho-physical strain, PRIW, and NA. The questionnaire was administered anonymously, and participants took part in the study voluntarily.

\subsection{Measures}

The following self-report measures were administered.

Participants were asked to rate their job performance using an item taken from the $\mathrm{Q}_{\mathrm{u}}$-Bo test, an instrument standardized for the Italian context (De Carlo, Falco, \& Capozza, 2008). The response scale ranged from 1 (very poor) to 10 (excellent).

Psycho-physical strain was assessed using a scale taken from the $\mathrm{Q}_{\mathrm{u}}$-Bo test (De Carlo et al., 2008). Respondents were asked to indicate how often, over the past two months, stress-related psychological and physical symptoms had appeared or exacerbated. Examples of scale items were "feeling tense and nervous" and "heartburn or pain in the stomach" for psychological and physical symptoms, respectively. The response scale ranged from 1 (never) to 6 (everyday). Cronbach's alpha for the overall scale was .92 .

PRIW was measured using an adaptation of the COVID-19 Perceived Risk Scale (CPRS; Yıldırım \& Güler, 2020) to the work context, that is, the CPRS-W (Falco, Girardi, Dal Corso, Yıldırım, \& Converso, 2021). As the original form of CPRS, the CPRS-W includes a cognitive dimension (e.g., "What is the likelihood that you would acquire the COVID-19 in your work organization?") and an emotional dimension (e.g., "How worried are you about a member of your work team contracting the COVID-19 in your work organization?") of personal risk. The five-point response scale ranged from 1 (negligible) to 5 (very large). In this study, the overall score of the CPRS-W was used as a measure of perceived risk related to COVID-19. Cronbach's alpha for the overall scale was .90 .

Negative affectivity was assessed using the Italian adaptation (Terracciano, McCrae, \& Costa, 2003) of the Positive and Negative Affect Schedule (PANAS; Watson, Clark, \& Tellegen, 1988). The scale is composed of ten items that describe different feelings and emotions (e.g., "irritable"). The response scale ranged from 1 (very slightly or not at all) to 5 (extremely). Cronbach's alpha for the overall scale was .88 .

\subsection{Data analysis}

The hypothesized relationships were tested using path analysis. In the estimated model, PRIW was the independent variable, psycho-physical strain was the mediator, and job performance was the dependent variable. Furthermore, NA moderated the relationship between PRIW and psycho-physical strain. If a significant interaction was found, then a simple slope analysis was conducted, to determine whether PRIW was associated with psycho-physical strain at high $(+1 S D)$ and low $(-1 S D)$ levels of NA (Preacher et al., 2007). The significance of the indirect effect at different levels of NA was then tested using the distribution of product method (Tofighi \& MacKinnon, 2011). To evaluate the goodness-of-fit of the models tested, the $\chi^{2}$ test was considered, as well as three additional fit indices, namely RMSEA, CFI, and SRMR. Values close to or smaller than .08 for RMSEA and SRMR, as well as values close to or greater than .90 for CFI, indicate an acceptable fit (Brown, 2015). The analyses were conducted using the lavaan package (Rosseel, 2012) for software R (R Core Team, 2020).

\section{Results}

The model showed a good fit to data: $\chi^{2}(4)=7.82, p=.10 ;$ RMSEA $=.052$, CFI $=.981$, SRMR $=.034$. Both PRIW $(\beta=.22, p<.001)$ and NA $(\beta=.78, p<.001)$ were positively associated with psychophysical strain, which, in its turn, was negatively associated with job performance $(\beta=-.21$, $p<.01)$. $\mathrm{H} 1$ and $\mathrm{H} 2$ were supported. The association between PRIW and job performance was not significant. Furthermore, NA moderated the association between PRIW and psycho-physical strain $(\beta=.14, p<.05)$. The association between PRIW and psycho-physical strain was positive and significant when NA was high $(\beta=.32, p<.001)$, but not significant when NA was low $(\beta=.11, n s)$. H3 was supported. Finally, the indirect effect of PRIW on job performance through psycho-physical strain was negative and significant when NA was high $(\beta=-.07,95 \%$ CI $-.13,-.02)$, but not significant when NA was low $(\beta=-.02,95 \% \mathrm{CI}-.06, .01)$. $\mathrm{H} 4$ was supported. 


\section{Discussion}

In line with the JD-R model, we proposed in this study that PRIW and NA can be conceptualized as a job and a personal demand, respectively. Accordingly, we hypothesized that PRIW is positively associated with psycho-physical strain, which, in its turn, is negatively associated with job performance. We also hypothesized that NA moderates the association between PRIW and psycho-physical strain, with this association being stronger when NA was high. Finally, we also hypothesized that the indirect effect of PRIW on job performance is moderated by NA.

The results of this study supported our predictions. PRIW was positively associated with psycho-physical strain and negatively associated with job performance, although indirectly in the latter case. Furthermore, NA exacerbated the positive association between PRIW and psycho-physical strain. Similarly, the indirect effect of PRIW on job performance was negative and significant when NA was high, but not significant when NA was low.

From a theoretical point of view, this study suggests that the JD-R can be successfully applied to safety at work during the ongoing outbreak of COVID-19. In this perspective, PRIW should be considered as a supplementary job demand for workers, who have to perform additional tasks to handle risky situations at work and to achieve their objectives safely (Leiter, 2005). In line with the health impairment process of the JD-R, this may have a negative impact on employees' health and job performance. Furthermore, this study suggests that high-NA employees may react more negatively to the perceived risk of infection at work, probably because NA is related to reduced coping abilities (Hofmann \& Kohlmann, 2019). Hence, individuals with high levels of NA may have an increased strain response to job demands, including PRIW (Spector, Zapf, Chen, \& Frese, 2000). Overall, at a more general level, the results of this study are consistent with transactional models of stress, which emphasize the interaction between situational and individual factors in the stress process (Ganster \& Rosen, 2013).

From a practical standpoint, this study suggests that organizations should provide workers with high levels of job resources to manage the risk of infection and achieve their work goals safely (i.e., primary prevention). Examples of resources are a good-quality communication about COVID-19 risks and rapid decision-making processes that prioritize safety at work (Falco et al., 2021). Furthermore, in terms of secondary prevention, interventions could help high-NA employees to develop skills to cope effectively with the risk of infection at work (e.g., problem- and meaning-focused coping strategies; Krok \& Zarzycka, 2020). Similarly, interventions could be aimed at fostering positive personal resources (e.g., self-efficacy and optimism).

\section{Conclusions}

Our study highlighted the importance of psychological safety at work during the ongoing outbreak of COVID-19. In a perspective of prevention and health promotion, it is important to consider those aspects of the job and the work environment that may negatively affect employees' well-being and performance, such as the perceived risk of being infected at work. Furthermore, by showing an interaction between job and personal demands, this study suggests that interventions should be tailored to the needs of individuals with different personality dispositions.

\section{References}

Bakker, A. B., \& Demerouti, E. (2017). Job demands-resources theory: Taking stock and looking forward. Journal of Occupational Health Psychology, 22, 273-285

Bolger, N., \& Zuckerman, A. (1995). A framework for studying personality in the stress process. Journal of Personality and Social Psychology, 69, 890-902.

Brown, T. A. (2015). Confirmatory factor analysis for applied research (2nd ed.). New York: Guilford Press.

Crawford, E. R., LePine, J. A., \& Rich, B. L. (2010). Linking job demands and resources to employee engagement and burnout: A theoretical extension and meta-analytic test. Journal of Applied Psychology, 95, 834-848.

De Carlo, A., Girardi, D., Falco, A., Dal Corso, L., \& Di Sipio, A. (2019). When does work interfere with teachers' private life? An application of the Job Demands-Resources model. Frontiers in Psychology, 10, 1121. 
De Carlo, N. A., Falco, A., \& Capozza, D. (2008). Test di valutazione del rischio stress lavoro-correlato nella prospettiva del benessere organizzativo, $Q_{u}$-Bo [Test for the assessment of work-related stress risk in the organizational well-being perspective, Qu-Bo]. Milano, Italy: FrancoAngeli.

Eaton, R. J., \& Bradley, G. (2008). The role of gender and negative affectivity in stressor appraisal and coping selection. International Journal of Stress Management, 15, 94-115

Falco, A., Girardi, D., Dal Corso, L., Yıldırım, M., \& Converso, D. (2021). The perceived risk of being infected at work: An application of the job demands-resources model to workplace safety during the COVID-19 outbreak. Manuscript submitted for publication.

Ganster, D. C., \& Rosen, C. C. (2013). Work stress and employee health: A multidisciplinary review. Journal of Management, 39, 1085-1122.

Hofmann, H., \& Kohlmann, C.-W. (2019). The role of positive and negative affectivity in healthy and unhealthy work-related behavior and experiences. European Journal of Health Psychology, 26, $56-67$.

Krok, D., \& Zarzycka, B. (2020). Risk perception of COVID-19, meaning-based resources and psychological well-being amongst healthcare personnel: The mediating role of coping. Journal of Clinical Medicine, 9, 3225

Leiter, M. P. (2005). Perception of risk: An organizational model of occupational risk, burnout, and physical symptoms. Anxiety, Stress, \& Coping, 18, 131-144.

Marek, J., Tangernes, B., \& Hellesøy, O. H. (1985). Experience of risk and safety. In O. H. Hellesøy (Ed.), Work environment Statfjord field. Oslo: Universitetsforlaget.

Nahrgang, J. D., Morgeson, F. P., \& Hofmann, D. A. (2011). Safety at work: A meta-analytic investigation of the link between job demands, job resources, burnout, engagement, and safety outcomes. Journal of Applied Psychology, 96, 71-94.

Preacher, K. J., Rucker, D. D., \& Hayes, A. F. (2007). Addressing moderated mediation hypotheses: Theory, methods, and prescriptions. Multivariate Behavioral Research, 42, 185-227

R Core Team. (2020). R: A Language and environment for statistical computing. Vienna, Austria: $\mathrm{R}$ Foundation for Statistical Computing.

Rosseel, Y. (2012). lavaan: An R package for structural equation modeling. Journal of Statistical Software, 48, 1-36

Rundmo, T., \& Iversen, H. (2004). Risk perception and driving behaviour among adolescents in two Norwegian counties before and after a traffic safety campaign. Safety Science, 42, 1-21.

Schaufeli, W. B., \& Bakker, A. B. (2004). Job demands, job resources, and their relationship with burnout and engagement: A multi-sample study. Journal of Organizational Behavior, 25, 293-315.

Spector, P. E., Zapf, D., Chen, P. Y., \& Frese, M. (2000). Why negative affectivity should not be controlled in job stress research: Don't throw out the baby with the bath water. Journal of Organizational Behavior, 21, 79-95

Terracciano, A., McCrae, R. R., \& Costa, P. T. (2003). Factorial and construct validity of the Italian Positive and Negative Affect Schedule (PANAS). European Journal of Psychological Assessment, 19, 131-141.

Tofighi, D., \& MacKinnon, D. P. (2011). RMediation: An R package for mediation analysis confidence intervals. Behavior Research Methods, 43, 692-700.

Watson, D., \& Clark, L. A. (1984). Negative affectivity: The disposition to experience aversive emotional states. Psychological Bulletin, 96, 465-490.

Watson, D., Clark, L. A., \& Tellegen, A. (1988). Development and validation of brief measures of positive and negative affect: The PANAS scales. Journal of Personality and Social Psychology, 54, 1063-1070.

Yaris, C., Ditchburn, G., Curtis, G. J., \& Brook, L. (2020). Combining physical and psychosocial safety: A comprehensive workplace safety model. Safety Science, 132, 104949.

Yıldırım, M., Güler, A. (2020). Factor analysis of the COVID-19 Perceived Risk Scale: A preliminary study. Death Studies. Advance online publication.

Zeijen, M. E. L., Brenninkmeijer, V., Peeters, M. C. W., \& Mastenbroek, N. J. J. M. (2021). Exploring the role of personal demands in the health-impairment process of the job demands-resources model: A study among master students. International Journal of Environmental Research and Public Health, 18, 632. 